\section{A topical approach to block HIV}

Topically applied microbicides can protect an individual from sexually acquiring an infection with HIV-1, and so could help to reduce the global spread of HIV-1. The development of these medications is complex, and several substances are being investigated at present. In a recent paper, published in Nature Medicine, Veazey and colleagues test whether virus transmission can be prevented by vaginal application of a monoclonal antibody specific for HIV-1 gp120.

In this study, the authors used simian-human immundeficiency virus (SHIV)-162P4, as this virus uses the chemokine receptor CCR5 to enter cells and so the results obtained should be applicable to human transmission of HIV-1. Initial experiments showed that $b 12$, a neutralizing human monoclonal antibody specific for HIV-1 gp120, could inhibit infection of macaque peripheral-blood mononuclear cells, immature human dendritic cells and human cervical explants in vitro.

To test its efficacy in vivo, rhesus macaque monkeys received b12 vaginally, either in a gel form or in saline, before being vaginally challenged with SHIV. Three of 12 monkeys that received $5 \mathrm{mg}$ of b12 became infected when they were challenged two hours after receiving the antibody, compared with 12 of 13 monkeys that received a control human monoclonal antibody. The authors tested lower doses of antibody but these were less effective, which indicates that the effect was dose dependent.

So, local application of b12 can protect monkeys from infection with SHIV through the vagina. These results indicate that there are positive prospects for the development of viral-entry inhibitors to prevent transmission of HIV in humans.

Jenny Buckland

(i) References and links

\section{ORIGINAL RESEARCH PAPER Veazey, R.S. et al.}

Prevention of virus transmission to macaque monkeys by vaginally applied monoclonal antibody to HIV-1 gp 120 . Nature Med. 10 February 2003 (DOl: 10.1038/nm833) FURTHER READING Stone, A. Microbicides: a new approach to preventing HIV and other sexually transmitted infections. Nature Rev. Drug Discov. 1, 977-985 (2002) WEB SITE

John Moore's lab: http://www.med.cornell.edu/research/ htmls/John\%20P.\%20Moore.html

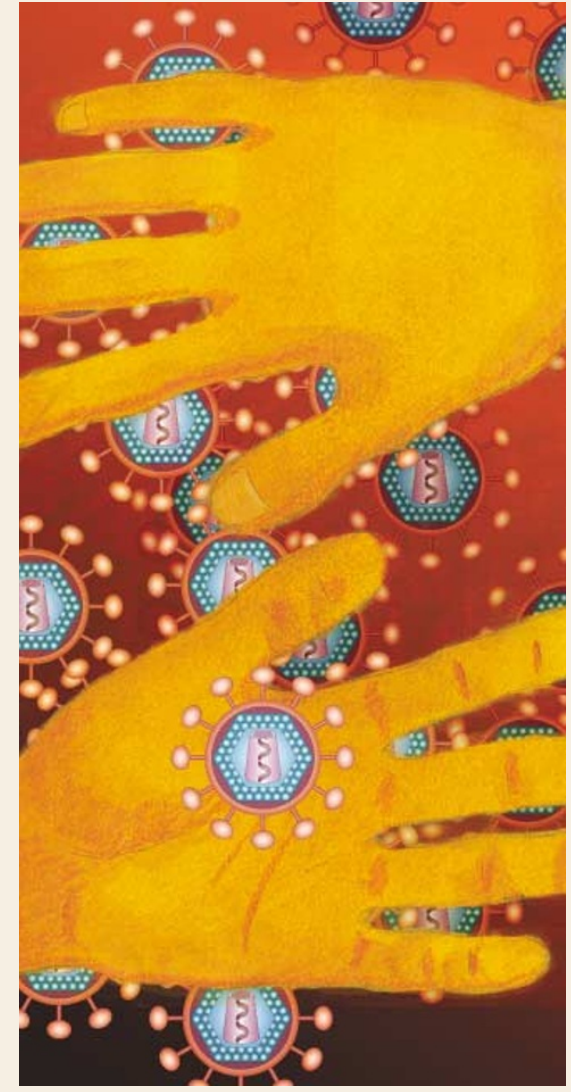

\section{T-CELL DEVELOPMENT}

\section{The right niche for Notch?}

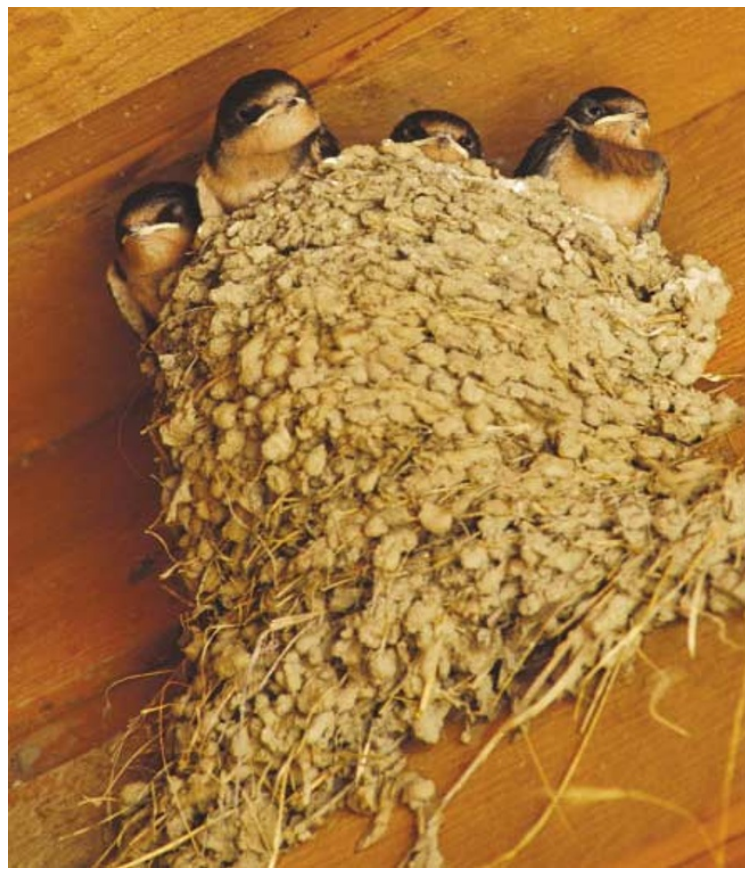

Although interactions between T-cell precursors and thymic stromal cells are known to be essential during thymocyte development, controversy exists over whether T-cell commitment occurs inside or outside the thymus. Similarly, recent studies have pointed to an important role for Notch1 during the initiation of T-cell development, but the timing of Notch signalling in relation to T-cell commitment is not known. Now, a study by Harman and colleagues published in The Journal of Immunology helps to answer these questions.

First, owing to the lack of suitable antibodies, the authors used reverse-transcriptase PCR to assess the expression of Notch1, and to look for evidence of Notch1 signalling by detecting expression of the Notch1 target genes hairy and enhancer of Split 1 (Hes1) and Deltex homologue 1 (Dtxl) in prethymic and intrathymic cells. Interleukin-7 receptor $\alpha$-chain (IL$7 \mathrm{R} \alpha)^{+} \mathrm{Lin}^{-}$lymphoid precursors from fetal liver expressed Notch1, but showed no evidence of Notch signalling, whereas intrathymic doublenegative (DN) $\mathrm{CD}^{-}{ }^{-} \mathrm{CD} 8^{-}$thymocytes expressed Notch1 and the Notch target genes. After removal from the thymic environment, however, the expression of Dtx 1 and Hes 1 by these DN cells decreased rapidly. Notch ligands (which are required for the activation of Notch) were shown to be expressed only by thymic epithelial cells (TECs), and not by lymphoid precursors from the thymus or fetal liver.

This indicates that the activation and maintenance of Notch signalling and function during thymocyte development depend on the entry of lymphoid progenitors into the thymus and their interaction with Notch ligands expressed by TECs. To investigate this further, Harman et al. looked at the activation of Notch during different stages of thymic colonization. IL-7R $\alpha^{+}$lymphoid progenitors from the perithymic mesenchyme (which have not yet contacted the thymic epithelium) expressed Notch1 and Dtxl, but not Hes1 or pT $\alpha$ (a component of the pre-T-cell receptor), whereas lymphoid progenitors recovered from areas of the thymic epithelium expressed all of these genes.

These findings support a role for Notch1 signalling in an intrathymic mechanism of T-cell commitment. As Notch1 can only be deleted from the DN stage onwards in currently available inducible $\mathrm{Notch}^{-/-}$mice, the relevance of these findings in vivo remains to be determined.

Jenny Buckland

(2) References and links

ORIGINAL RESEARCH PAPER Harman, B. C. etal. Entry into

the thymic microenvironment triggers Notch activation in the earliest migrant T-cell progenitors. J. Immunol. 170, 1299-1303 (2003) 